\title{
Evaluation of a Murine Model for Intranasal and Intraperitoneal Infection by Haemophilus parasuis: Short Report
}

\author{
Ana-Judith Martín de la Fuente, César-B. Gutiérrez Martín*, Sonia Martínez Martínez, \\ Rafael Frandoloso and Elías-F. Rodríguez Ferri
}

\begin{abstract}
Department of Animal Health, Section of Microbiology and Immunology, Faculty of Veterinary Medicine, University of León, 24007-León, Spain
\end{abstract}

\begin{abstract}
NMRI mice were evaluated as model for experimental infection by Haemophilus parasuis by intranasal (IN) and intraperitoneal (IP) routes. No deaths, clinical signs and macroscopic lesions were noticed after receiving several doses (except when the mice were infected intraperitoneally with the highest dose), and the organism was cleared rapidly from spleen and lungs. These results suggest that NMRI mice are not a suitable model to reproduce experimentally Glässer's disease.
\end{abstract}

Keywords: Haemophilus parasuis, Glässer's disease, mouse, experimental model.

\section{INTRODUCTION}

Haemophilus parasuis, a non-haemolytic, nicotinamide adenine dinucleotide (NAD)-dependent Gram-negative rod belonging to the family Pasteurellaceae, is a commensal of the upper respiratory tract of healthy pigs [1]. However, it is also considered an important porcine pathogen and the etiological agent of Glässer's disease, which is characterized by fibrinous polyserositis, polyarthritis and meningitis. This disease is an emerging challenge in the pig-rearing industry worldwide today, often associated with significant financial losses [2]. In addition, $H$. parasuis is also related to septicemia, penumonia and myositis [3].

Experimental studies in the natural host are difficult because $H$. parasuis-free pigs are required. Caesarean-derived, colostrum-deprived (CDCD) piglets have been used successfully, but this model requires intense management, entailing a high cost. Both naturally farrowed, colostrum-deprived (NFCD) pigs $[4,5]$ and guinea pigs $[6,7]$ have been proposed to reproduce the disease, with lower costs and less handling cares. Although the mouse is not a natural host for H. parasuis, an alternative experimental model to pigs and guinea pigs for Glässer's disease, easy to be managed, would be of a great interest. In this respect, not many studies about $H$. parasuis infections of mice have been performed, showing contradictory results depending on the medium in which this organism was suspended [6].

The goal of this study was to examine the ability of Nagasaki strain of $H$. parasuis serotype 5 (considered highly virulent in pigs) to cause in mice a disease similar to that found in the natural host by intranasal (IN) and intraperitoneal (IP) routes, and thus to investigate the feasibility of using experimental infection of the mouse as a cost-effective alternative model of Glässer's disease for further virulence and protection studies.

*Address correspondence to this author at the Department of Animal Health, Section of Microbiology and Immunology, Faculty of Veterinary Medicine, University of León, 24007-León, Spain; Tel: 34987291 203; Fax: 34987291 304; E-mail: dsacgm@unileon.es

\section{MATERIALS \& METHODOLOGY}

The Nagasaki reference strain of serotype 5 (one of the most prevalent serotypes worldwide [1]) of $H$. parasuis was grown on chocolate-blood agar plates (bioMérieux, Barcelona, Spain), and incubated at $37^{\circ} \mathrm{C}$ for $24-48 \mathrm{~h}$ in an atmosphere containing $5 \% \mathrm{CO}_{2}$. Bacterial cells were harvested in sterile saline, centrifuged, and resuspended in RPMI 1640 medium (Sigma-Aldrich, Madrid, Spain). In order to standardize the number of cells inoculated per animal, a viable count (colony-forming units -CFU-) of the suspension was determined by plating dilutions onto chocolate-blood agar and incubating overnight at $37^{\circ} \mathrm{C}$. The cells were kept at $4^{\circ} \mathrm{C}$ up to the time of inoculation. A new viable count was carried out after it in order to check the inoculum viability and to discard contaminations. Similar counts were recorded before and after inoculation and other bacteria than $H$. parasuis were not isolated.

A total of 80 female NMRI mice (weighing 15 to $20 \mathrm{~g}$ ) were housed in a controlled animal facility $\left(22 \pm 2^{\circ} \mathrm{C}, 12-\mathrm{h}\right.$ light/12-h dark cycle), allowed food and water ad libitum, and acclimated no less than 7 days before starting of the experiment. All animal handling and procedures were approved by the University of León Ethical Committee and conducted under a licence, in accordance with the conditions approved by the Spanish Government. Mice were randomly assigned into 16 groups of five animals. Seven groups were inoculated in the nasal cavity and seven others were injected intraperitoneally. Ten-fold doses (from $2 \times 10^{3}$ to $2 \times 10^{9} \mathrm{CFU}$ per mouse) were given in each inoculation route, one to each of the groups separated. Two other groups (one for each route), which remained as infection controls, were given RPMI alone instead of $H$. parasuis cells. Mice were anesthetized with isoflurane $\left(\right.$ Forano $^{\circledR}$, Abbott Laboratories, Madrid, Spain) before inoculation. For intranasal (IN) infection, a volume of $50 \mu \mathrm{l}$ of bacterial suspension was dropped onto the external nares, and for intraperitoneal (IP) injection, 200 $\mu 1$ were aseptically injected in the abdominal cavity. At five days post-inoculation, all surviving mice were sacrified under terminal anaesthesia. 
Clinical signs were recorded every hour for the first day and then daily until death or euthanasia. The number of dead mice was recorded and the $\mathrm{LD}_{50}$ was calculated by the method of Reed and Muench [8]. All surviving animals were necropsied and macroscopic lesions recorded. Samples were collected aseptically from the spleen, lungs, abdominal and thoracic cavities for bacteriological examination. Individual organs were ground in sterile homogenisers with $5 \mathrm{ml}$ of RPMI, centrifuged and $100 \mu 1$ of the supernatant were plated onto chocolate-blood agar. The suspected colonies were studied for NAD requirement by observing the satellite phenomenon on blood agar cross-streaked with a nurse strain of Staphylococcus intermedius.

For the bacterial elimination assays, 21 female NMRI mice were inoculated by the IN route with $2 \times 10^{9} \mathrm{CFU}$ of $H$. parasuis suspended in RPMI. Three mice were sacrificed at $0,4,6,8,10,12$ and 24 hours after the exposure, and their lungs and spleen were removed for bacterial reisolation and identification as described previously. In addition, $100 \mu \mathrm{l}$ of each supernatant were inoculated onto chocolate-blood agar plates in duplicate to determine the number of CFU.

\section{RESULTS AND DISCUSSION}

All mice inoculated by the IN route survived after the infection, and only those receiving the highest dose $\left(2 \times 10^{9}\right.$ $\mathrm{CFU}$ ) showed tremor or ruffled coat within $24 \mathrm{~h}$ after bacterial exposure (Table 1). None of the mice in the groups treated with lower doses and in the control group showed clinical signs. No remarkable macroscopic lesions were found at the necropsy in any group of mice, and $H$. parasuis could not be recovered from any of the 40 animals infected by this route when they were necropsied five days after infection. It was not possible to calculate the $\mathrm{LD}_{50}$ for the IN route because of the absence of mortality in any of the seven doses inoculated.

The IN route, through which $H$. parasuis preferentially infects the natural host [1], has hardly been assessed in mice. From our results, it can be concluded that NMRI mice were not susceptible to Nagasaki strain when they were inoculated intranasally, even using doses as high as $2 \times 10^{9} \mathrm{CFU}$. However, similar or lower doses have been proved to develop severe Glässer's disease (and even death rapidly after exposure) in CDCD [9] and NFCD [4] pigs by this route, as well as in conventional guinea pigs [7] and SPF pigs [10] inoculated intratracheally.

Concerning the results of the bacterial elimination assays, $H$. parasuis could be isolated in high numbers (more than 300 colonies) from the lungs of each of the three mice ne- cropsied at 4, 6, 8 and $10 \mathrm{~h}$ post-inoculation, but the count decreased considerably $(36.0 \pm 12.7 \mathrm{CFU}) 12 \mathrm{~h}$ after exposure and the organism was only recovered from two of the three mice in this group. H. parasuis was completely eliminated from the lungs after $12 \mathrm{~h}$ following the IN inoculation of $2 \times 10^{9} \mathrm{CFU}$ of Nagasaki strain. On the other hand, $H$. parasuis colonized the spleen between 8 and $10 \mathrm{~h}$ postinfection, reaching substantially lower counts $(28.5 \pm 9.2$ and $37.0 \pm 4.2$, respectively) than in lungs at the same time. The organism was cleared from the spleen after $10 \mathrm{~h}$.

These results indicate that, although $H$. parasuis is capable to gain access to murine lungs and spleen after IN exposure, it is rapidly eliminated from both organs and, therefore, is unable to induce disease. One possible explanation might be the activation of the innate immunity, presumably through phagocytosis. This mechanism would have been able to effectively destroy $H$. parasuis before $24 \mathrm{~h}$ following inoculation. However, the use of mice strain with specific mutations of the innate immune system, such as the toll-like receptors, might have provided different results. It would be also speculated that the rapid bacterial clearance might be related to the inability of this organism of acquiring iron from murine transferrin, because this high-affinity iron uptake system seems to be rather specific for the natural host [11].

All mice infected intraperitoneally with $2 \times 10^{9} \mathrm{CFU}$ of $H$. parasuis died 6 to $24 \mathrm{~h}$ following inoculation, with dullness, tremor, closed eyes, ruffled coat, and a severe congestion in the abdominal cavity (Table 1). In contrast, the mice inoculated with doses lower than $2 \times 10^{9} \mathrm{CFU}$ or with RPMI alone survived for all the exposure time, without clinical signs and gross lesions. $H$. parasuis was only recovered from the abdominal cavities of the five mice receiving $2 \times 10^{9}$ CFU. Other bacteria than $H$. parasuis were not isolated from these animals. For the IP route, the $\mathrm{LD}_{50}$ of Nagasaki strain was of $6.32 \times 10^{8} \mathrm{CFU}$.

Quite different to our results, other authors [6] observed no deaths among the mice inoculated intraperitoneally with the same infecting dose of strain no. 4 (belonging to serotype 1) suspended in physiological saline, with only unspecific clinical signs, no gross lesions and $H$. parasuis recovery from only one mouse; however, $58.3 \%$ of mice died when the same dose was suspended in a broth containing NAD instead of saline, and the recovery rate increased to $50 \%$.

\section{CONCLUSION}

In spite of being pathogenic for mice by IP inoculation with $2 \times 10^{9} \mathrm{CFU}$ of $H$. parasuis in our study and being recovered from all animals inoculated with this dose, the ab-

Table 1. Results of Experimental Infection of Mice with Different Doses of H. parasuis, Strain Nagasaki (Serotype 5) by Intranasal (IN) or Intraperitoneal (IP) Inoculations

\begin{tabular}{|c|c|c|c|c|c|c|}
\hline Dose (CFU) & Route & $\begin{array}{c}\text { No. of } \\
\text { Mice }\end{array}$ & $\begin{array}{c}\text { No. of } \\
\text { Dead Mice }\end{array}$ & $\begin{array}{c}\mathbf{N}^{\mathbf{o}} \text {. of Mice with } \\
\text { Clinical Signs }\end{array}$ & $\begin{array}{c}\mathbf{N}^{\mathbf{0}} \text { of Mice } \\
\text { with Lesions }\end{array}$ & $\begin{array}{c}\mathbf{N}^{\mathbf{0}} \text { of Mice from which H. parasuis } \\
\text { was Recovered }\end{array}$ \\
\hline \hline $\begin{array}{c}2 \times 10^{3}, 2 \times 10^{4}, 2 \times 10^{5}, \\
2 \times 10^{6}, 2 \times 10^{7} \text { and } 2 \times 10^{8}\end{array}$ & IN & 5 & 0 & 0 & 0 & 0 \\
\hline $2 \times 10^{9}$ & IP & 5 & 0 & 0 & 0 & 0 \\
& IN & 5 & 0 & 5 & 5 & 5 \\
\hline
\end{tabular}


sence of clinical signs and macroscopic lesions resembling those produced in natural cases of disease strongly suggest that modeling Glässer's disease caused by Nagasaki strain of $H$. parasuis in NMRI mice is not a viable alternative to use of pigs and guinea pigs.

\section{ACKNOWLEDGEMENTS}

This work was supported by grant AGL2005-01210/ GAN from the Spanish Ministry of Education and Science. A.J.M.F. was a recipient of a long-term predoctoral fellowship from this Spanish Ministry.

\section{REFERENCES}

[1] Oliveira S, Pijoan C. Haemophilus parasuis new trends on diagnosis, epidemiology and control. Vet Microbiol 2004; 99: 1-12.

[2] Nielsen R. Pathogenicity and immunity studies of Haemophilus parasuis serotypes. Acta Vet Scand 1993; 34: 193-198.

[3] Hoefling DC. The various forms of Haemophilus parasuis. Swine Health Prod 1991; 2: 1.

[4] Oliveira S, Pijoan C, Galina L, Blanco I, Canals A. Evaluation of naturally farrowed colostrums-deprived pigs as a model for ex- perimental infection by Haemphilus parasuis. Can Vet J 2003; 67: 146-150.

[5] Blanco I, Galina-Pantoja L, Oliveira S, Pijoan C, Sánchez C, Canals A. Comparison between Haemophilus parasuis infection in colostrums-deprived and sow reared piglets. Vet Microbiol 2004; 103: 21-27.

[6] Morozumi T, Hiramune T, Kobayashi K. Experimental infections of mice and guinea pigs with Haemophilus parasuis. Nat Inst Anim Health Q 1982; 22: 23-31.

[7] Rapp-Gabrielson VJ, Gabrielson DA, Schramber GJ. Comparative virulence of Haemophilus parasuis serovars 1 to 7 in guinea pigs. Am J Vet Res 1992; 54: 997-1004.

[8] Reed LJ, Muench HA. A simple method of estimating fifty per cent endpoints. Am J Trop Med Hyg 1938; 27: 493-497.

[9] Vahle JL, Haynes JS, Andrews JJ. Experimental reproduction of Haemophilus parasuis infection in swine: clinical, bacteriologic and morphologic findings. J Vet Diagn Invest 1995; 7: 476-480.

[10] Amano H, Shibata M, Takahasihi K, Sasaki Y. Effects on endotoxin pahtogenicity in pigs with acute septicemia of Haemophilus parasuis infection. J Vet Med Sci 1997; 59: 451-455.

[11] Del Río ML, Gutiérrez Martín CB, Rodríguez-Barbosa JI, Navas J, Rodríguez Ferri EF. Identification and characterization of the TonB region and its role in transferrin-mediated iron acquisition in Haemophilus parasuis. FEMS Immunol Med Microbiol 2005; 45: 7586. 